\title{
Intimal Sarcoma of the Pulmonary Artery, Case Report
}

\author{
Emad M. Barsoum, Ashraf M. Habib, Nouran M. Roby \\ Barsoum Oncology Clinic, Cairo, Egypt \\ Email: nouran.elroby@gmail.com
}

How to cite this paper: Barsoum, E.M., Habib, A.M. and Roby, N.M. (2021) Intimal Sarcoma of the Pulmonary Artery, Case Report. Open Access Library Journal, 8: e7225.

https://doi.org/10.4236/oalib.1107225

Received: February 6, 2021

Accepted: April 13, 2021

Published: April 16, 2021

Copyright $\odot 2021$ by author(s) and Open Access Library Inc.

This work is licensed under the Creative

Commons Attribution International

License (CC BY 4.0).

http://creativecommons.org/licenses/by/4.0/

\begin{abstract}
Intimal sarcoma is a rare type of mesenchymal tumor that arises from the intima of large and small vessels. Most commonly it arises from the pulmonary artey or the thoracic aorta. We report a case diagnosed with right pulmonary artery intimal sarcoma after CT guided biopsy. The patient underwent surgical excision of the tumor successfully without residual and no post-operative complications, then received adjuvant chemotherapy. Patient was disease free till her last follow up in 12/2020. Conclusion: As there is no standard of care for the intimal sarcoma, it was treated by extrapolating data from sarcoma guidelines. Complete surgical excision will remain the best prognostic factor for the survival.
\end{abstract}

\section{Subject Areas}

Oncology

\section{Keywords}

Sarcoma, Pulmonary Artery, Case Report

\section{Introduction}

Vascular neoplasms are classified as leiomyiosarcomas, angiosarcomas and intimal sarcomas and immunohistochemistry is needed to differentiate between them [1].

Intimal sarcomas are rare and lethal malignant tumors that typically affect larger vessels: the aorta, inferior vena cava, and pulmonary arteries [2].

Intimal sarcomas have a greater prevalence in the middle aged females [1].

It can be misdiagnosed as a pulmonary embolism, mediastinal mass, or tumor emboli, leading to delay in the appropriate treatment, such as prolonged anti- 
coagulation or thrombolysis [3].

Its prognosis is very poor based on the reported cases [2].

The key to the survival is the early and accurate diagnosis, to help in early effective treatment [3].

There is no established standard of care for it. As reported, surgical options for PA sarcomas vary from palliative stenting, debulking, and endarterectomy to excision with or without pneumonectomy and with or without reconstruction of the PA [4].

Here, we report the case of a 49 -year-old woman with pulmonary artery intimal sarcoma.

\section{Case Report}

A 49-year-old female patient presented with progressive course of cough and hemoptysis of 4 months duration. CT scan for chest without contrast on 25/5/2017 which showed well defined complex density lesion, $5.2 \times 4.7 \times 7 \mathrm{~cm}$, intrapulmonary at posterior segment of lower lobe, extending to ipsilateral hilum.

Further investigations using PET/CT scan with IV contrast showed metabolically active right pulmonary neoplasm (lobulated mass extending from hilum to lung periphery) measuring $5.4 \mathrm{~cm}$ with SUVmax uptake of 6.3 .

The case was discussed in a multidisciplinary team (MDT) and was advised for CT guided biopsy. The biopsy was done without any complications and pathology showed malignant round cell tumor and immunophenotyping confirmed the diagnosis of intimal sarcoma.

The Patient underwent right lower lobectomy and lymph nodes sampling in July 2017.

Pathology showed low grade sarcoma compatible with intimal sarcoma, free pleural margins, free bronchial margins, hilar lymph node, Subcarinal lymph node were involved, 3 Interlobar lymph nodes were free from infiltration (Lymph node score 2/5).

The patient has received 4 cycles of adjuvant chemotherapy Ifosfamide Doxorubicin a 3 days protocol ended on 5/10/2017 with good tolerance and accepted toxicity. Then she was kept under follow-up with CT scan and clinical examination and she was disease free till her last follow up on 24/12/2020.

\section{Discussion}

PAS has a very low incidence and only few hundreds cases have been described in the literature [5].

Commonly age of presentation is 45 - 55 years with a female to male ratio of $2: 1[6]$.

The etiology and risk factors of this tumor have not yet been clarified but it has been shown that it may be related to the CDK4 gene mutation [7].

Complete surgical excision remains the mainstay of treatment as it improves 
the survival [8].

In our reported case, the main challenge was to ensure the complete resection with negative margin and that was after assessing the respectability using PET-CT and CT.

There is limited information on the role of chemotherapy and radiotherapy in the treatment of this disease [4].

Due to the rarity of that type of sarcoma, data extrapolated from the sarcoma clinical trial recommended adjuvant chemotherapy following surgery.

Chemotherapy regimens that have been used include Adriamycin plus isosfamide, gemcitabine plus taxane or dacerbazine regimens [4].

Xu et al., described a case which was treated with chemotherapy upfront using triplet therapy of doxorubicin, cisplatin, and ifosfamide for two cycles. Because of intolerable side effects, she received vinorelbine and cisplatin for the next four cycles with remarkable regression of the tumor then she was oral cyclophosphamide (50 $\mathrm{mg}$ t.i.d) as a maintenance therapy [9].

Our patient has received doublet chemotherapy regimen using IfosfamideDoxorubicin with very good tolerance and accepted toxicity

Hirose et al. also offered chemotherapy followed by radiation to their patient has developed recurrence after 2 months of complete surgical resection [3].

After reviewing this case, in the intimal sarcoma, respectability should be assessed before going through the surgery. Offering the patient adjuvant treatment is must after the surgery due to the rarity and poor prognosis of that type of tumors.

\section{Conclusion}

In conclusion, there is no standard of care for treatment for the intimal sarcoma. Complete surgical resection offers the best option with longer survival. More studies should be conducted to offer more information for diagnosis and treatment.

\section{Consent}

We report that we obtained an informed consent from the patient for article submission and publication.

\section{Conflicts of Interest}

The authors declare no conflicts of interest regarding the publication of this paper.

\section{References}

[1] López-Gómez, J., Flores-Vázqueza, E.R., Salazar-Álvareza, Ma.A., et al. (2017) Treatment of Intimal Sarcoma of Peripheral Veins. International Journal of Surgery Case Reports, 31, 93-98. https://doi.org/10.1016/j.ijscr.2017.01.018

[2] Moritz, A.T. (1923) About Primary Re-Formations of the Heart. Virchow's Archive 
of Pathological Anatomy and Physiology and Clinical Medicine, 245, 43-54.

[3] Takashi, H., et al. (2009) A Case of Intimal Sarcoma of the Pulmonary Artery Treated with Chemoradiotherapy. Internal Medicine, 48, 245-249.

[4] Blackmon, S.H., Rice, D.C., Correa, A.M., et al. (2009) Management of Primary Pulmonary Artery Sarcomas. The Annals of Thoracic Surgery, 87, 977-984. https://doi.org/10.1016/j.athoracsur.2008.08.018

[5] Mussot, S., Ghigna, M.-R., Mercier, O., Fabre, D., et al. (2013) Retrospective Institutional Study of 31 Patients Treated for Pulmonary Artery Sarcoma. European Journal of Cardio-Thoracic Surgery, 43, 787-793.

https://doi.org/10.1093/ejcts/ezs387

[6] Gan, H.-L., Zhang, J.-Q., Huang, X.-Y. and Yu, W. (2013) The Wall Eclipsing Sign on Pulmonary Artery Computed Tomography Angiography Is Pathognomonic for Pulmonary Artery Sarcoma. PLoS ONE, 8, e83200.

https://doi.org/10.1371/journal.pone.0083200

[7] Feng, G.A.O., et al. (2009) Novel Mutation of the Cyclin-Dependent Kinase 4 Gene in a Chinese Patient with Intimal Sarcoma of the Pulmonary Artery. Chinese Medical Journal, 122, 1107-1109.

[8] Hoiczyk, M., Iliodromitis, K., Erbel, R., et al. (2012) Intimal Sarcoma of the Pulmonary Artery with Unusual Findings: A Case Report. Clinical Research in Cardiology, 101, 397-401. https://doi.org/10.1007/s00392-012-0425-5

[9] Xu, Y.J., Wang, K., Geng, Y.T., Shao, Y.F and Yin, Y.M. (2012) A Case of Intimal Sarcoma of the Pulmonary Artery Successfully Treated with Chemotherapy. International Journal of Clinical Oncology, 17, 522-527.

https://doi.org/10.1007/s10147-011-0338-8 